\title{
Cache-Enabled Device to Device Networks with Aloha Based Multimedia Delivery
}

\author{
Xiaoshi Song, Chao Jia, Jiacheng Wang, Xile Shen, Ning Ye, and Weimin Lei \\ School of Computer Science and Engineering, Northeastern University \\ Shenyang, China \\ songxiaoshi@cse.neu.edu.cn
}

\begin{abstract}
In this paper, we study the performance of large-scale cacheenabled device to device (D2D) networks. The mobile helper$\mathrm{s}(\mathrm{MHs})$ and the user equipments(UEs) are modeled as two independent homogeneous Poisson pointprocess (HPPP). The UEs can get file from MHs which are assumed to have caching capabilities within the collaboration distance of D2D transmission. An Aloha type of multimedia delivery protocol is considered, under which the available MHs make independent decisions to launch the transmissions with probability $p_{m}$. According to stochastic geometry, we derive and analyze the transmission probability of MHs . And then, the coverage probability of the randomly requested files is characterized. Next, we work out the successful content delivery probability of the cache-enabled D2D network. By applying the obtained results of successful content delivery probability, we optimize the probabilistic caching strategy of MHs. Particularly, under the proposed Aloha based multimedia delivery protocol, to maximize the successful content delivery probability of the cache-enabled D2D network, each $\mathrm{MH}$ should simply cache the most popular file. Simulations are included to demonstrate our analysis.
\end{abstract}

\section{KEYWORDS}

Cache-enabled D2D networks, Aloha based multimedia delivery protocol, optimal probabilistic caching strategy, stochastic geometry, successful content delivery probability.

\section{INTRODUCTION}

Driven by the ever-increasing demand of bandwidth-intensive multimedia services, due to the proliferation of smart mobile devices growing exponentially, data traffic boomed over recent years. The mobile data traffic is expected to reach 49 exabytes per month by 2021, an sevenfold increase over 2016, in which multimedia streaming contribute more than three-fourths percent, predicted by Cisco [4]. In response to such a rapid

Permission to make digital or hard copies of all or part of this work for personal or classroom use is granted without fee provided that copies are not made or distributed for profit or commercial advantage and that copies bear this notice and the full citation on the first page. To copy otherwise, to republish, to post on servers or to redistribute to lists, requires prior specific permission and/or a fee.

Mobimedia 2017, July 13-14, Chongqing, People's Republic of China Copyright (๑) 2017 EAI 978-1-63190-156-0 the multimedia contents closer to users, but also simultaneously the spatial recuse and (coded or uncoded) multicasting opportunities in data dissemination are utilized to reduce the heavy pressure of the fast growing traffic with caching ability enabled at the nearest mobile helpers.

In order to study cache-enabled device-to-device network$\mathrm{s}$, it is necessary to apply tools from stochastic geometry $[2,3,10-12,19]$ for tractable characterization of key network performance metrics, such as coverage, spatial throughput, and so on. In particularly, In [11], aiming at maximizing the average density of successful receptions under different fading distributions, Malak et al. developed the optimal spatiallyindependent content caching strategies. In [12], Malak et al. further researched the optimal geographic content placement problem for device-to-device networks, and put forward spatially correlated caching strategies by maximizing the device-to-device cache hit probability. In [2], for different degrees of node mobility, Jarray et al. discussed the hit performance of caching in device-to-device networks. In [3], Chen et al. offered analytical and numerical results to compare caching at small cells and the performance of caching at mobile devices, based on the density of cache-served requests, average power consumption and the cache hit probability. In [19], analyzing a closed-form approximation of cache-aided throughput, Chen et al. further investigated the optimal caching probabilities with numerical optimization. In [10], Afshang et al. developed a comprehensive analytical framework with foundations in stochastic geometry to describe the performance of cluster-centric content placement in a cache-enabled device-to-device network

In this paper, different from that in [2, 3, 10-12, 19], to study the performance of large-scale cache-enabled device-todevice networks, we develop a framework of analysis which mobile helpers (MHs) and user equipments (UEs) follow homogeneous Poisson point process (HPPP)distribution. An Aloha type multimedia delivery protocol is considered, under which an available $\mathrm{MH}^{1}$ decides to launch the transmission with probability $p_{m}$. Besides, we assume that the popularity of the multimedia files obeys a Zipf distribution. By making use of tools from stochastic geometry, we derive the transmission probability of MHs with the Aloha based multimedia delivery protocol. Then, We describe the coverage probability of the randomly requested files. Next, The successful content delivery probability is characterized. According to the obtained results of successful content delivery probability,

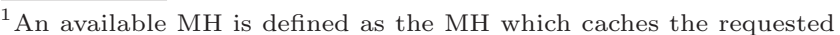
files.
} 


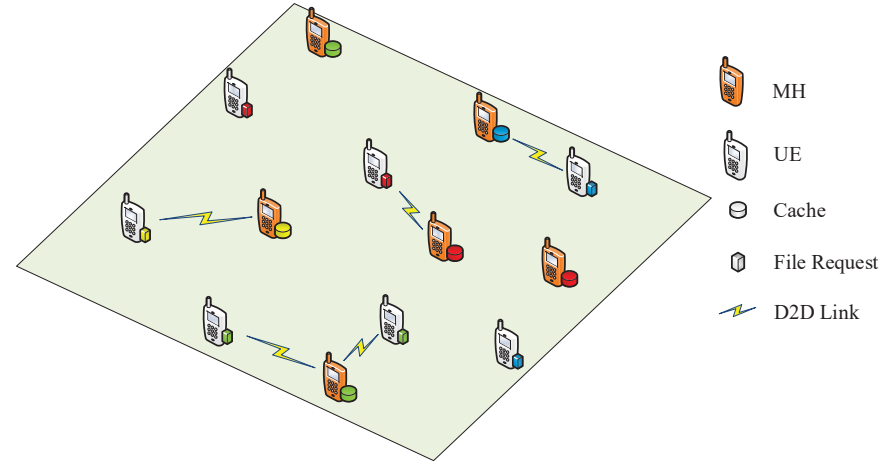

Figure 1: Cache-enabled device to device network formed by MHs, UEs, and a library of multimedia files $\mathcal{F}$.

we optimize the probabilistic caching strategy of MHs. Especially, we find each $\mathrm{MH}$ caching the most popular file can maximize the successful content delivery probability. Finally, we validate our analysis through simulations.

The remainder of this paper is organized as follows. In Section II, the system model is introduced. In Section III, we derive the transmission probability of MHs. The successful content delivery performance of is given in Section IV. In Section V, We discuss the optimal probabilistic caching strategy. Section VI provides simulation results to demonstrate the validity of our proposals. Finally, Section VII conclude the paper .

\section{SYSTEM MODEL}

We consider a large-scale cache-enabled device-to-device network formed by dedicated MHs, intended UEs, and a library of multimedia files $\mathcal{F}:=\{1,2, \cdots, F\}$ on $\mathbb{R}^{2}$ as illustrated in Fig. 1. We model the locations of MHs and UEs as two independent HPPPs with density $\lambda_{m}$ and $\lambda_{u}$, respectively. To simplify the analysis, the files in library $\mathcal{F}$ is assumed to be of the same size, and each $\mathrm{MH}$ possess a cache memory of only 1 file $(M=1)$. The $\mathrm{MH}$ caches the $f$-th file randomly in $\mathcal{F}$ with probability $c_{f}$, which is decentralized probabilistic caching strategy. The popularity of the $f$-th file in $\mathcal{F}$ is assumed to follow the Zipf distribution as

$$
p_{f}=\frac{1 / f^{\gamma}}{\Sigma_{j=1}^{F} 1 / j^{\gamma}},
$$

where $\gamma \geq 0$ denotes the Zipf parameter.

The model of propagation channel is the combination of the large-scale path-loss and the small-scale Rayleigh fading given by

$$
g(d)=h d^{-\alpha},
$$

where $d$ denotes the propagation distance, $h$ denotes the exponentially distributed power coefficient with unit mean, and $\alpha$ denotes the path-loss exponent. To do this simply, we ignore the thermal noise in the regime of interest and simply focus on the received signal to interference ratio (SIR). The transmit power of MHs is denoted by $P_{d}$. Let $R_{d}$ denote the collaboration distance of device-to-device transmission between $\mathrm{MH}$ and UE. $\theta_{d}$ is defined as the SIR target for successful data receptions under device-to-device communication.

An Aloha type multimedia delivery protocol is considered, under which an available $\mathrm{MH}$ makes independent decisions to launch the transmission with probability $p_{m}$. Then, upon finding the active MHs, the UEs simply associate with the nearest ones for data transmissions. Further, it is assumed that each MH may simultaneously serve multiple UEs with the same content request in the vicinity.

\section{TRANSMISSION PROBABILITY}

In this section, under the Aloha based multimedia delivery protocol. Particularly, we characterize the transmission probability of MHs in large-scale cache-enabled device-to-device networks. To derive the transmission probability, we define $\zeta_{d}$ as the probability that a $\mathrm{MH}$ is under request. At first, we deduce $\zeta_{d}$ in the following lemma.

Lemma 3.1. For large-scale cache-enabled device-to-device networks, the probability that a $M H$ is under request is given by

$$
\zeta_{d}=\sum_{f=1}^{F} c_{f} \cdot\left(1-e^{-\lambda_{u} p_{f} \pi R_{d}^{2}}\right) .
$$

Proof. Given $p_{f}, c_{f}$, and $R_{d}$, we can consider the void probability that The tagged MH doesn't get request of the file which it has cached within a distance of $R_{d}$, which completes the proof of Lemma 3.1.

Based on Lemma 3.1, we prepare for evaluating the transmission probability of MHs under the Aloha based multimedia delivery protocol, as given by following theorem.

TheOREM 3.2. For large-scale cache-enabled device-todevice networks, the transmission probability of $M H s$ under the Aloha based multimedia delivery protocol is given by

$$
q_{d}=\zeta_{d} \cdot p_{m} .
$$

Proof. With Lemma 3.1, under the Aloha based multimedia delivery protocol, (4) is immediately obtained. This thus completes the proof of Theorem 3.2.

We define $\Psi_{m}^{a}$ as the point process formed by active MHs with the Aloha based multimedia delivery protocol and define $\lambda_{m}^{a}$ as the corresponding density. According to Theorem 3.2, we have the following corollary.

Corollary 3.3. For large-scale cache-enabled device-todevice networks with the Aloha based multimedia delivery protocol, the density of $\Psi_{m}^{a}$ is given by

$$
\lambda_{m}^{a}=\lambda_{m} \cdot q_{d}
$$

We paying attention to that the requests of UEs are spatially correlated for MHs within a distance of $R_{d}$. Hence, $\Psi_{m}^{a}$ does't follow a HPPP. Besides, since the higher order 
statistics of $\Psi_{m}^{a}$ are tricky, the successful content delivery probability of the cache-enabled device-to-device networks, which bases on the probability generating functional (PGFL) [9] of $\Psi_{m}^{a}$, is hard to be described exactly. To work out this difficulty, similar to $[9,16,18]$, we make the following approximation on $\Psi_{m}^{a}$, which will be proved later by simulations in Section VI.

Conjecture 3.4. For large-scale cache-enabled device-todevice networks, $\Psi_{m}^{a}$ follows a HPPP with density $\lambda_{m}^{a}$.

In the following section, with Theorem 3.2 and Assumption 3.4, it will be characterized that the successful content delivery performance of large-scale cache-enabled device-todevice networks with the Aloha based multimedia delivery protocol.

\section{SUCCESSFUL CONTENT DELIVERY PROBABILITY}

Thanks to the stationarity of the point processes composed by the MHs and UEs, we concentrates on a typical UE at the origin denoted by $\mathbf{U}$ with its related $\mathrm{MH}$ at a random distance of $d_{f}$ away denoted by $\mathbf{M}$, where the distribution of $d_{f}$ depends on the popularity of file $f$, to analyze the successful content delivery performance of the large-scale cache-enabled device-to-device networks. Then, under Assumption 3.4, by Slivnyak's theorem [9], the locations of the rest of the active MHs follow a HPPP with density $\lambda_{m}^{a}$. Based on this fact, in the following theorem, the successful content delivery probability of the cache-enabled device-to-device network is characterized.

THEOREM 4.1. For large-scale cache-enabled device-todevice networks, with the Aloha based multimedia delivery protocol, according to Assumption 3.4, the successful content delivery probability is expressed as

$$
\begin{aligned}
\tau_{d}= & \sum_{f=1}^{F} p_{f} \int_{0}^{R_{d}} \exp \left\{-\left(1-c_{f}\right) \int_{0}^{\infty} \eta(u) u \mathrm{du}\right\} \\
& \times \exp \left\{-c_{f} \int_{d_{f}}^{\infty} \eta(u) u \mathrm{du}\right\} \cdot \varpi_{f}\left(d_{f}\right) \mathrm{dd}_{\mathrm{f}},
\end{aligned}
$$

where

$$
\eta(u)=\frac{2 \pi \lambda_{m}^{a}}{1+\frac{u^{\alpha}}{\theta_{d} d_{f}^{\alpha}}},
$$

and

$$
\varpi_{f}\left(d_{f}\right)=2 \lambda_{m}^{a} c_{f} \pi d_{f} \cdot e^{-\lambda_{m}^{a} c_{f} \pi d_{f}^{2}} .
$$

Proof. The proof is omitted due to the space limitation.

\section{PROBABILISTIC CACHING PLACEMENT}

In this section, we aim at maximizing the successful content delivery probability $\tau_{d}$ of the cache-enabled device-to-device networks with the Aloha based multimedia delivery protocol by finding the optimal solutions of $c_{f}$. In particular, according to Theorem 4.1, the optimization problem is formulated as

$$
\begin{aligned}
(\mathrm{P} 1): & \max _{c_{f}} \tau_{d} \\
\text { s.t. } & \sum_{f=1}^{F} c_{f} \leq 1, \\
& c_{f} \geq 0 .
\end{aligned}
$$

We find the expression of $\tau_{d}$ complex and the coupling effect between $\lambda_{m}^{a}$ and $c_{f}$, so it is hard to obtain an exact characterization of the optimal solutions $\tilde{c}_{f}^{*}$ of $(\mathrm{P} 1)$. To work out this trouble, by applying that $\tau_{d}$ is an increasing function in regards to $\lambda_{m}^{a}$ (which can be numerically proved) and thereby $\zeta_{d}$, we consider an replaceable optimization problem as

$$
\begin{aligned}
(\mathrm{P} 2): & \max _{c_{f}} \zeta_{d} \\
\text { s.t. } & \sum_{f=1}^{F} c_{f} \leq 1, \\
& c_{f} \geq 0 .
\end{aligned}
$$

According to (3), we deduce the optimal solution $c_{f}^{*}$ of (P2) in the following lemma.

Lemma 5.1. The optimal solution $c_{f}^{*}$ of $(\mathrm{P} 2)$ is given by

$$
\begin{aligned}
& c_{1}^{*}=1 \\
& c_{i}^{*}=0, i=2,3, \ldots, F,
\end{aligned}
$$

i.e., to maximize $\zeta_{d}$ we can cache the most popular file in $\mathcal{F}$.

Proof. It can be easily proved that (P2) is a linear programming problem in regards to $c_{f}$. So by applying simplex method [17] on (P2), we can immediately obtain (15). This thus completes the proof of Lemma 5.1.

\section{NUMERICAL RESULTS}

In this section, we present simulation results on the performance of the studied large-scale cache-enabled device-todevice network with Aloha based multimedia delivery protocol to validate our analytical results. Throughout this section, unless specified otherwise, we set $P_{d} / N_{d}=20, \theta_{d}=1$, $R_{d}=15, \gamma=1, \alpha=4, c_{f}=p_{f}, p_{m}=0.9, F=5$, and $\lambda_{m}=0.005$.

\subsection{Transmission Probability}

Fig. 2 compares the analytical and simulated results on the transmission probability $q_{d}$ versus the density of UEs $\lambda_{u}$. It is shown in the picture that the transmission probability $q_{d}$ of MHs is an increasing function with respect to $\lambda_{u}$ (and thereby $\zeta_{d}$ ), which is visually expected according to Theorem 3.2.

\subsection{Successful Content Delivery Probability}

Fig. 3 reveals the successful content delivery probability $\tau_{d}$ versus the density of UEs $\lambda_{u}$. Several observations are in order. Firstly, it is shown that $\tau_{d}$ is an increasing function of $\lambda_{u}$ (and thereby $\zeta_{d}, q_{d}$, and $\lambda_{m}^{a}$ ), which is consistent with 


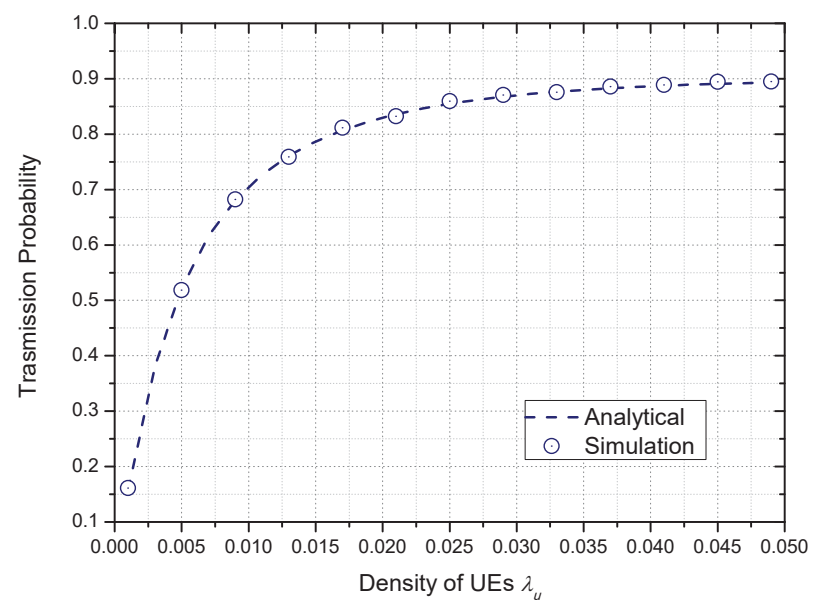

Figure 2: Transmission probability $q_{d}$ versus the density of UEs $\lambda_{u}$.

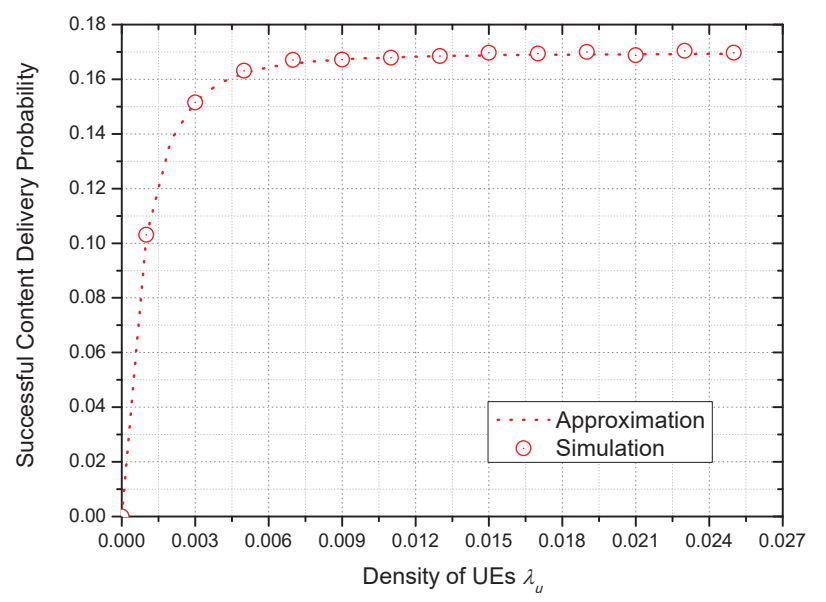

Figure 3: Successful content delivery probability $\tau_{d}$ versus the density of UEs $\lambda_{u}$.

the monotonicity of $\xi_{f}$ but varied from that of $\mathcal{C}_{f}$. Secondly, we derive the approximated successful content delivery probability $\tau_{d}$ in Theorems 4.1 which is quite accurate. An direct explanation of the above observation is that, as mentioned in [18] and [8], the higher-order statistics of $\Psi_{m}^{a}$ have a marginal effect on the computed Laplace transform of the aggregate interference from all active $\mathrm{MH}$ (except the typical $\mathrm{MH}$ ) to the typical UE at the origin.

\subsection{Optimal Probabilistic Caching Placement}

In the end, Fig. 4 confirms the optimality of the mentioned content caching strategy for $\gamma=1$. In particular, we compare the performance of the optimal content caching strategy with the even caching strategy (where the MHs independently cache the files in $\mathcal{F}$ with even probabilities) and that of the

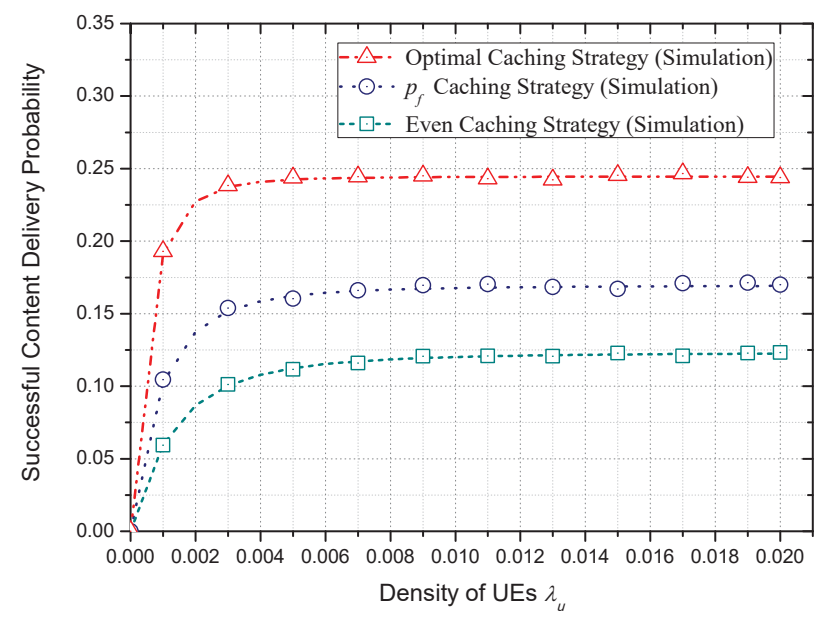

Figure 4: Comparison of the successful content delivery performance.

$p_{f}$ caching strategy (where the MHs independently cache the $f$-th file in $\mathcal{F}$ with probability $p_{f}$ ) as far as successful content delivery probability. We can observe from Fig. 4 that the successful content delivery performance of the proposed content caching strategy overweighs that of the even caching strategy and the $p_{f}$ caching strategy, which is expected.

\section{CONCLUSION}

In this paper, we have studied the performance of large-scale cache-enabled device-to-device networks under Aloha based multimedia delivery protocol. By using tools from stochastic geometry, we characterize the transmission probability of MHs and the successful content delivery probability of the cacheenabled device-to-device network. According to the obtained results of successful content delivery probability, we work out the optimal probabilistic caching strategy of MHs. In particular, with the Aloha based multimedia delivery protocol, we suggest to simply cache the most popular file at the MHs for the sake of maximizing the successful content delivery probability of the cache-enabled device-to-device network. Simulations has been supplied for validating our analysis. It is hoped that the results in this paper will offer new insights the practical design of large-scale cache-enabled device-todevice networks.

\section{ACKNOWLEDGMENTS}

This work is supported by the Fundamental Research Funds for the Central Universities under Grant No. N150403001, the Major Research Project of Northeastern University in 2017 under Grant No. ZD1714, and the National Natural Science Foundation of China under Grant 61671141, 61401079.

\section{REFERENCES}

[1] Z. Han W. Chen B. Bai, L. Wang and T. Svensson. 2016. Caching based socially-aware D2D communications in wireless content delivery networks: a hypergraph framework. IEEE Wireless Commun 23, 4 (Aug. 2016), 74-81. 2016. 
[2] J. Chedia and A. Giovanidis. 2016. The effects of mobility on the hit performance of cached D2D networks. in Proc. Int. Symp. on Modeling and Optimization in Mobile, Ad Hoc, and Wireless Networks, Tempe, Arizona (May 2016).

[3] Z. Chen and M. Kountouris. 2016. D2D caching vs. small cell caching: where to cache content in a wireless network. In Proc. IEEE Int. Workshop on Signal Processing Advances in Wireless Communications, Edinburgh, UK.

[4] Cisco. 2017. Cisco visual networking index: Global mobile data traffic forecast update, 2016-2021. Whitepaper (June 2017).

[5] C. Yang D. Liu, B. Chen and A. F. Molisch. 2016. Caching at the wireless edge: design aspects, challenges, and future directions. IEEE Commun 54, 9 (Sept. 2016), 22-28.

[6] X. Tian X. Wang H. Liu, Z. Chen and M. Tao. 2014. On contentcentric wireless delivery networks. IEEE Wireless Commun 21 , 6 (Dec. 2014), 118-125.

[7] Z. Chen H. Liu and L. Qian. 2016. The three primary colors of mobile systems. IEEE Commun.Mag 54, 9 (Sept. 2016), 15-21.

[8] M. Haenggi and R. K. Ganti. 2009. Interference in Large Wireless Networks. Foundations and Trends in Networking.

[9] C. Lee and M. Haenggi. 2012. Interference and outage in Poisson cognitive networks. IEEE Trans. Wireless Commun 11, 4 (April 2012), 1392-1401.

[10] H. S. Dhillon M. Afshang and P. H. J. Chong. 2015. Fundamentals of clustercentric content placement in cache-enabled device-todevice networks. IEEE Transactions on Communications. 64,6 (June 2015), 2511-2526.

[11] M. Al-Shalash M. Derya and J. G. Andrews. 2016. Optimizing the spatial content caching distribution for device-to-device communications. arXiv:1609.00419 (2016).

[12] M. Al-Shalash M. Derya and J. G. Andrews. 2016. Spatially correlated content caching for device-to-device communications. arXiv:1609.00419 (2016)

[13] G. Caire M. Ji and A. F. Molisch. 2016. Fundamental limits of caching in wireless D2D networks. IEEE Trans. Inf. Theory 62 2 (Feb. 2016), 849-869.

[14] J. Liu M. Sheng, C. Xu and J. Song. 2016. Enhancement for content delivery with proximity communications in caching enabled wireless networks: architecture and challenges. IEEE Commun Mag 54, 8 (Aug. 2016), 70-76. 2016.

[15] A. G. Dimakis N. Golrezaei, A. F. Molisch and G. Caire. 2013 Femtocaching and device-to-device collaboration: A new architecture for wireless video distribution. IEEE Commun.Mag 51, 4 (April 2013), 142-149.

[16] T. Nguyen and F. Baccelli. 2010. A probabilistic model of carrier sensing based cognitive radio. in Proc. of IEEE Symposium on New Frontiers in Dynamic Spectrum Access Networks, Singapore (April 2010).

[17] R. J. Vanderbei. 2008. Linar Programming: Foundations and Extensions, Third Edition. Springer.

[18] D. Liu X. Song, C. Yin and R. Zhang. 2014. Spatial throughput characterization in cognitive radio networks with threshold-based opportunistic spectrum access. IEEE J. Sel. Areas Commun 32 , 11 (Nov. 2014)

[19] N. Pappas Z. Chen and M. Kountouris. 2016. Probabilistic caching in wireless D2D networks: cache hit optimal vs. throughput optimal. IEEE Commun. Letters (2016).

[20] Z. Ding W. Wang Z. Zhao, M. Peng and H. V. Poor. 2016. Cluster content caching: an energy-efficient approach to improve quality of service in cloud radio access networks. IEEE J. Sel. Areas Commun 34, 5 (May 2016), 1207-1221. 